University of Wollongong

Research Online

Faculty of Informatics - Papers (Archive)

Faculty of Engineering and Information

Sciences

2010

\title{
Phase error correction based on Inverse Function Shift Estimation in Phase Shifting Profilometry using a digital video projector
}

Yang Liu

University of Wollongong

Jiangtao Xi

University of Wollongong, jiangtao@uow.edu.au

Yanguang Yu

University of Wollongong, yanguang@uow.edu.au

Joe F. Chicharo

University of Wollongong, chicharo@uow.edu.au

Follow this and additional works at: https://ro.uow.edu.au/infopapers

Part of the Physical Sciences and Mathematics Commons

\section{Recommended Citation}

Liu, Yang; Xi, Jiangtao; Yu, Yanguang; and Chicharo, Joe F.: Phase error correction based on Inverse Function Shift Estimation in Phase Shifting Profilometry using a digital video projector 2010.

https://ro.uow.edu.au/infopapers/3544

Research Online is the open access institutional repository for the University of Wollongong. For further information contact the UOW Library: research-pubs@uow.edu.au 


\title{
Phase error correction based on Inverse Function Shift Estimation in Phase Shifting Profilometry using a digital video projector
}

\author{
Abstract \\ Fringe Pattern Profilometry (FPP) is 3D surface measuring technique based on triangulation. The \\ utilization of digital projection in FPP system introduces significant phase distortion for Phase Shifting \\ Profilometry (PSP), because of the nonlinear response of digital video projectors, which is referred as \\ gamma distortion. Considering that the distorted phase has a stable function for a reference plane, this \\ paper proposes an approach based on inverse function shift estimation (IFSE) to detect the spatial shift \\ of the distorted phase caused by object height. This spatial shift is independent of projector's gamma \\ distortion and accurate surface can be reconstructed based it. The simulation results show that the \\ proposed method can almost completely eliminate gamma distortion in reconstructed surface and we \\ obtain more than 5 times improvement in practical experiments.

\section{Disciplines} \\ Physical Sciences and Mathematics

\section{Publication Details} \\ Liu, Y., Xi, J., Yu, Y. \& Chicharo, J. F. (2010). Phase error correction based on Inverse Function Shift \\ Estimation in Phase Shifting Profilometry using a digital video projector. Optical Metrology and Inspection \\ for Industrial Applications (pp. 78550W-1-78550W-8). USA: SPIE.
}




\title{
Phase error correction based on Inverse Function Shift Estimation in Phase Shifting Profilometry using a digital video projector
}

\author{
Yang Liu, Jiangtao Xi*, Yanguang Yu and Joe Chicharo \\ School of Electrical, Computer and Telecommunications Engineering, \\ University of Wollongong, Northfields Ave, Wollongong, NSW, 2522
}

\begin{abstract}
Fringe Pattern Profilometry (FPP) is 3D surface measuring technique based on triangulation. The utilization of digital projection in FPP system introduces significant phase distortion for Phase Shifting Profilometry (PSP), because of the nonlinear response of digital video projectors, which is referred as gamma distortion. Considering that the distorted phase has a stable function for a reference plane, this paper proposes an approach based on inverse function shift estimation (IFSE) to detect the spatial shift of the distorted phase caused by object height. This spatial shift is independent of projector's gamma distortion and accurate surface can be reconstructed based it. The simulation results show that the proposed method can almost completely eliminate gamma distortion in reconstructed surface and we obtain more than 5 times improvement in practical experiments.
\end{abstract}

\section{INTRODUCTION}

Optical noncontact surface measuring has been widely used in industrial archaeology, machine vision, mechanical engineering, etc. Fringe Pattern Profilometry (FPP) is one of the most popular measuring methods and has been extensively studied and various approaches are developed, such as Fourier Transform Profilometry (FTP) ${ }^{1-2}$ Phase Shifting Profilometry (PSP), ${ }^{3-4}$ Spatial Phase Detection (SPD) ${ }^{5}$ and Inverse Function Shift Estimation (IFSE) ${ }^{6}$ Among them PSP is the most widely employed one due to its high accuracy and immunity to background illumination and variable reflectivity. However, it requires the projected fringe to be sinusoidal, otherwise errors arise.

With the recent development of digital projection technology, traditional optical approaches, such as optical gratings and interference, have been replaced by digital projection to generate fringe patterns. Especially for PSP approaches, digital projection has a few key advantages, such as precise shifting control and fast projection. However, digital projectors are nonlinear devices which have an inherent characteristic referred as gamma distortion. Due to the Gamma distortion, it is difficult to produce precise sinusoidal patterns, and distinct errors are aroused when using PSP. Previously, a lot of work has been done to reduce such errors. Baker et al. ${ }^{7}$ employed neural network to train waveform of captured images, but the method proposed increases the measuring time significantly. Huang et al. ${ }^{8}$ proposed a double three-step PSP to eliminate gamma nonlinearity, which requires multiple images required. Zhang ${ }^{9-10}$ proposed a Look Up Table (LUT) method to compensate gamma nonlinearity error, but the generation of a LUT requires an extra calibration board, which make the whole measuring procedure complicated and not automatic. Liu et al. ${ }^{11}$ established a mathematical Gamma model, based on which phase correction methods were proposed to improve accuracy. However, Liu's Gamma model is derived without consideration of ambient light interferences.

As an alternate effort to reduce the nonlinear distortion, $\mathrm{Hu}$ et al. proposed a IFSE $^{6}$ based on Generalized Analysis Model $(\mathrm{GAM})^{12}$. GAM describes the fringe deformed by the object is a shifted version of that on the reference. By substituting the intensity value of objects into the inverse function of reference plane, the corresponding spatial shift will be obtained. And IFSE is one approach to build the inverse function required in GAM. Accordingly, surface can be

* jiangtao@uow.edu.au

Optical Metrology and Inspection for Industrial Applications, edited by Kevin Harding, Peisen S. Huang, Toru Yoshizawa, Proc. of SPIE Vol. 7855, 78550W · @ 2010 SPIE · CCC code: 0277-786X/10/\$18 · doi: 10.1117/12.870369 
reconstructed by implementation IFSE on intensity images directly. Different form PSP, results of this approach are not affected by gamma distortion and only one image is required. However, another two negative factors are not considered in this approach ${ }^{6}$; ambient light and reflectivity difference between reference plane and object. This severely restricts the application range of IFSE.

When examining the distorted phase by PSP, it is not randomly varying but has a certain function. Motivated by this, we find the distorted phase satisfies the conditions of GAM. Therefore, we propose to apply IFSE calculating spatial shift between distorted phases, just after unwrapping the phase by PSP algorithm. Due to the features of IFSE, hight based on spatial shift is not impacted by the nonlinear gamma response of video projectors.

This paper is organized as follows. In section 2, we explained the mathematic background of the proposed method and how phase shift is calculated. In section 3, we give simulation and experimental results to confirm the correctness of the proposed method. Section 4 concludes this paper.

\section{PRINCIPLES}

\subsection{Three-step Phase Shifting Profilometry}

Usually, FPP use a system setup as shown in Figure 1. A fringe pattern, denoted as $u(x, y)$ is projected along $\overrightarrow{C O}$. For the pattern only varying with respect to the coordinate $x$ and remaining constant on $y$ direction, for the sake of simplicity, coordinate $y$ is ignore in following deducing and we use $u(x)$ denoting the fringe light intensity on a reference plane by the projector:

$$
u(x)=1+\cos (2 \pi f x)
$$

where $f$ is the spatial frequency of the fringe on the reference plane. In the presence of an object, from the camera's view, the projected fringe have a spatial shift alone $x$ direction, which is demonstrated as distance $\overline{A B}$ in Figure 1 . We refer the spatial shift as $\delta(x)$. Hence the fringe turn out to be $u(x-\delta(x))$.

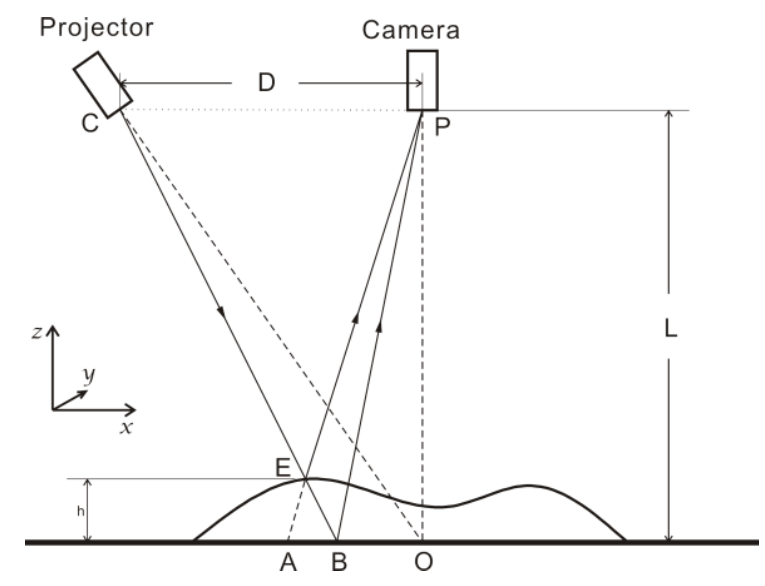

Figure 1. Optical system setup

Due to $\triangle C P E$ and $\triangle A B E$ are similar in, the fringe shift caused by object surface height $h(x)$ can be represented as: 


$$
h(x)=\frac{L \delta(x)}{D+\delta(x)}
$$

where $D$ and $L$ are the system parameter shown in Figure 1. To carry out PSP, three images are necessary and fringes projected are required to be sinusoidal. With consideration of projector's response $f_{c}(u)$, surface reflectivity $A(x)$ and background illumination $B(x)$, the $n$th image intensity is as follows:

$$
\begin{aligned}
& s_{n}(x)=A(x) f_{c}\left(u\left(x+\frac{n}{3 f}\right)\right)+B(x) \\
& d_{n}(x)=A(x) f_{c}\left(u\left(x-\delta(x)+\frac{n}{3 f}\right)\right)+B(x)
\end{aligned}
$$

Ideally, the projector's response is supposed to be linear; for simplicity $f_{c}(u)=u$. Then, we can retrieve the fringe sinusoid phase from intensity images using three-step PSP $^{3}$ :

$$
\varphi(x)=-\arctan \frac{\sum_{n=1}^{3} I_{n}(x) \sin (2 \pi n / N)}{\sum_{n=1}^{3} I_{n}(x) \cos (2 \pi n / N)}
$$

Note that the coordinate $x$ range within one period of sinusoid function in this paper, because phase wrapping problem is not considered here.

When replace $I_{n}(x)$ with $s_{n}(x)$ and $d_{n}(x)$, we obtain the fringe phase for the reference plane, $\varphi_{\text {ref }}(x)$, and object surface, $\varphi_{o b j}(x)$, respectively:

$$
\begin{aligned}
& \varphi_{r e f}(x)=2 \pi f x \\
& \varphi_{o b j}(x)=2 \pi f[x-\delta(x)]=2 \pi f x-\Delta \varphi(x)
\end{aligned}
$$

where $\Delta \varphi(x)=2 \pi f \delta(x)$ and is the phase shift by object surface height. Then we can derive the object's height by combining Eqs. (2) and (5):

$$
h(x)=\frac{\Delta \varphi(x) L}{2 \pi f_{0} D-\Delta \varphi(x)}
$$

\subsection{Gamma distortion and shift detection}

As mentioned above, most digital video projectors are associated with gamma distortion, and their response function modelled as a power function with a single parameter follows:

$$
f_{c}(u)=u^{\gamma}
$$

where $\gamma$ is a positive number and usually $1<\gamma<3$. Combining Eqs. (3) and (7) we have: 


$$
\begin{aligned}
& s_{n}(x)=A(x)\left(u\left(x+\frac{n}{3 f}\right)\right)^{\gamma}+B(x) \\
& d_{n}(x)=A(x)\left(u\left(x-\delta(x)+\frac{n}{3 f}\right)\right)^{\gamma}+B(x), 2 \text { and } 3
\end{aligned}
$$

The nonlinear response causes a change of the projected fringes. This also results in distortions in the phases extracted by three-step PSP in Eq.(4). Let us use $\phi(x)$ to denote the distorted phase. We can also obtain $\phi_{r e f}(x)$ and $\phi_{o b j}(x)$ by substituting Eqs. (8) into Eq. (4). But the difference between them, $\Delta \phi(x)=\phi_{r e f}(x)-\phi_{o b j}(x)$, cannot be used in Eq. (6). Otherwise, notable errors are aroused as shown in Figure 2.
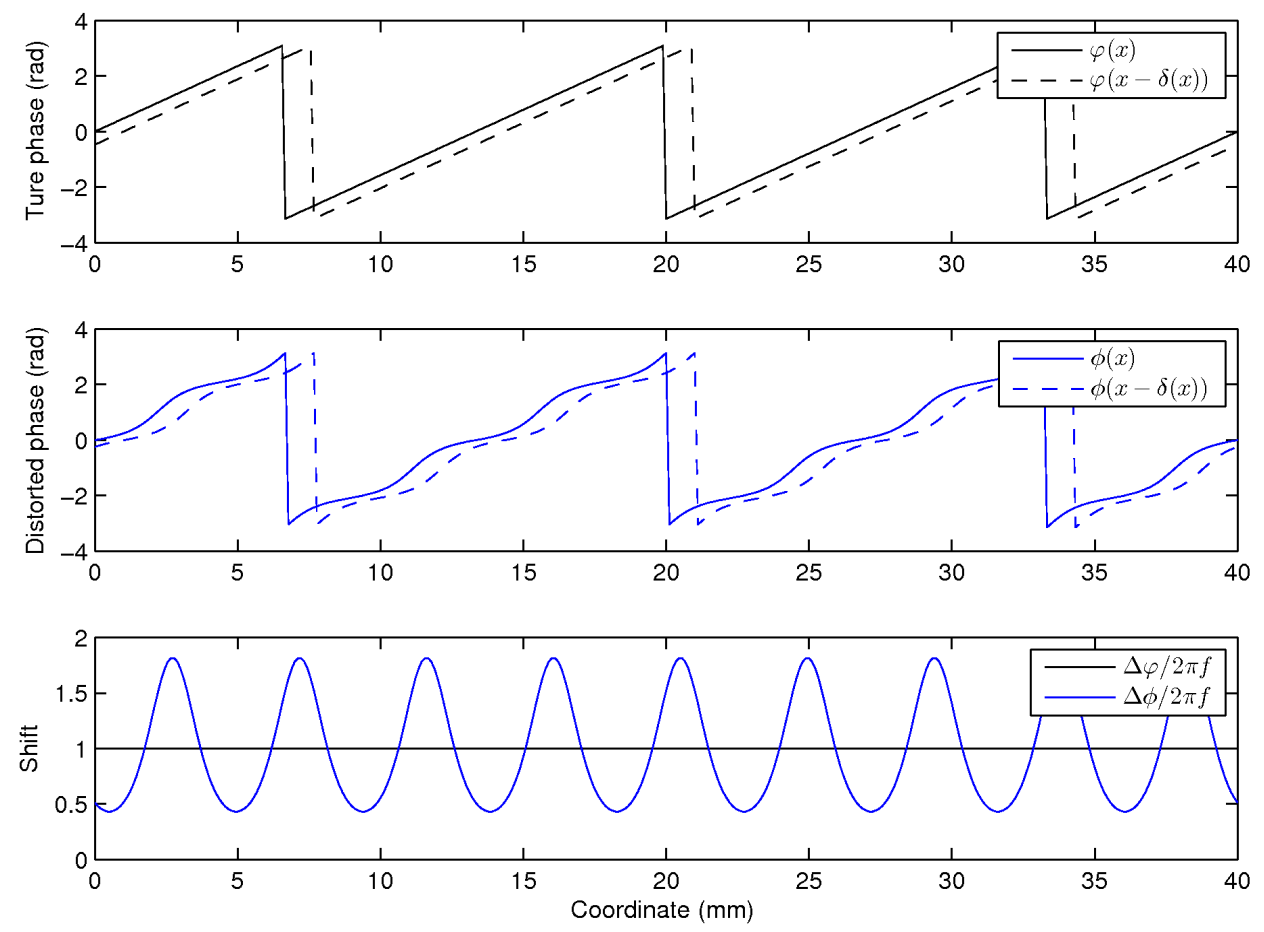

Figure 2. Derivation of ground truth and distorted phase calculated by 3 -step PSP when $\gamma=2.33$ and $\delta(x)=1$. Up:

Original phase $\varphi(x)$ and shifted phase $\varphi(x-\delta(x))$. Mid: Distorted phase $\phi(x)$ and shifted one $\phi(x-\delta(x))$.

Down: Shift $\delta(x)$ and calculated value in traditional approaches

To avoid gamma distortion in PSP, we propose a method to obtain $\delta(x)$. Combining Eqs. (4) and (8), we have distorted phase of the reference and denote it as $F_{r e f}(x)$ :

$$
F_{r e f}(x)=-\arctan \frac{\sqrt{3}\left\{[1+\cos (2 \pi f x+2 \pi / 3)]^{\gamma}-[1+\cos (2 \pi f x+4 \pi / 3)]^{\gamma}\right\}}{2[1+\cos (2 \pi f x)]^{\gamma}-[1+\cos (2 \pi f x+2 \pi / 3)]^{\gamma}-[1+\cos (2 \pi f x+4 \pi / 3)]^{\gamma}}
$$

Similarly, the distorted phase of objects:

$$
F_{o b j}(x)=-\arctan \frac{\sqrt{3}\left\{[1+\cos (2 \pi f x-\Delta \varphi(x)+2 \pi / 3)]^{\gamma}-[1+\cos (2 \pi f x-\Delta \varphi(x)+4 \pi / 3)]^{\gamma}\right\}}{2[1+\cos (2 \pi f x-\Delta \varphi(x))]^{\gamma}-[1+\cos (2 \pi f x-\Delta \varphi(x)+2 \pi / 3)]^{\gamma}-[1+\cos (2 \pi f x-\Delta \varphi(x)+4 \pi / 3)]^{\gamma}}
$$


Because $A(x)$ and $B(x)$ are all cancelled in Eq. (4), $F_{o b j}(x)$ satisfies the following equation.

$$
F_{o b j}(x)=F_{r e f}(x-\delta(x))
$$

This just meets the description in GAM by $\mathrm{Hu}^{12}$. Once having the inverse function of $F_{r e f}(x)$, we can determine the spatial shift, which is related to surface height, using the following equation.

$$
\delta(x)=x-F_{r e f}^{-1}\left(F_{o b j}(x)\right)
$$

We employ polynomial curve fitting to establish $F_{r e f}^{-1}(x)$. This process is described as following.

1. Curve fitting starts from a low order, say $k=1$ and a maximum error $e_{m}$ is provided at first.

2. Interchange distorted phase samples $F_{r e f}(x)$ and its corresponding coordinate $x$. Based on this two set of data, implement least squares curve fitting at $k$ th order. A set of coefficients, $C_{k}$ in Eq.(13), can be obtained.

3. Continue to step 4 , once the fitted curve satisfying a preset error $e_{m}$. Otherwise, $k=k+1$ and repeat step 2 and step 3.

4. The whole procedure returns a set of fitting polynomial coefficients, which stores the objective function $F_{r e f}^{-1}(x)$.

$$
F^{-1}(x)=C_{0}+C_{1} x+C_{2} x^{2}+\cdots+C_{k} x^{k} \pm e_{m}
$$

With an established inverse function and phase maps from three-step PSP, we can retrieve shift $\delta(x)$ by substituting the distorted phase values calculated by three-step PSP into Eq.(12). Height of the object based on $\delta(x)$ as Eq.(6) does not suffer from gamma distortion.

\section{SIMULATION AND EXPERIMENT}

In order to compare the performance of proposed method against traditional PSP, we carried out simulations on both of them. A triangular shape shift is added to a gamma distorted sinusoidal signal, and its phase is extracted by three-step PSP algorithm as shown in Figure 3. Results of the proposed approach and traditional PSP are also given in Figure 3. After applying IFSE, root mean square error (RMSE) of phase shift by PSP has declined from 0.1949 to 0.0008 . 

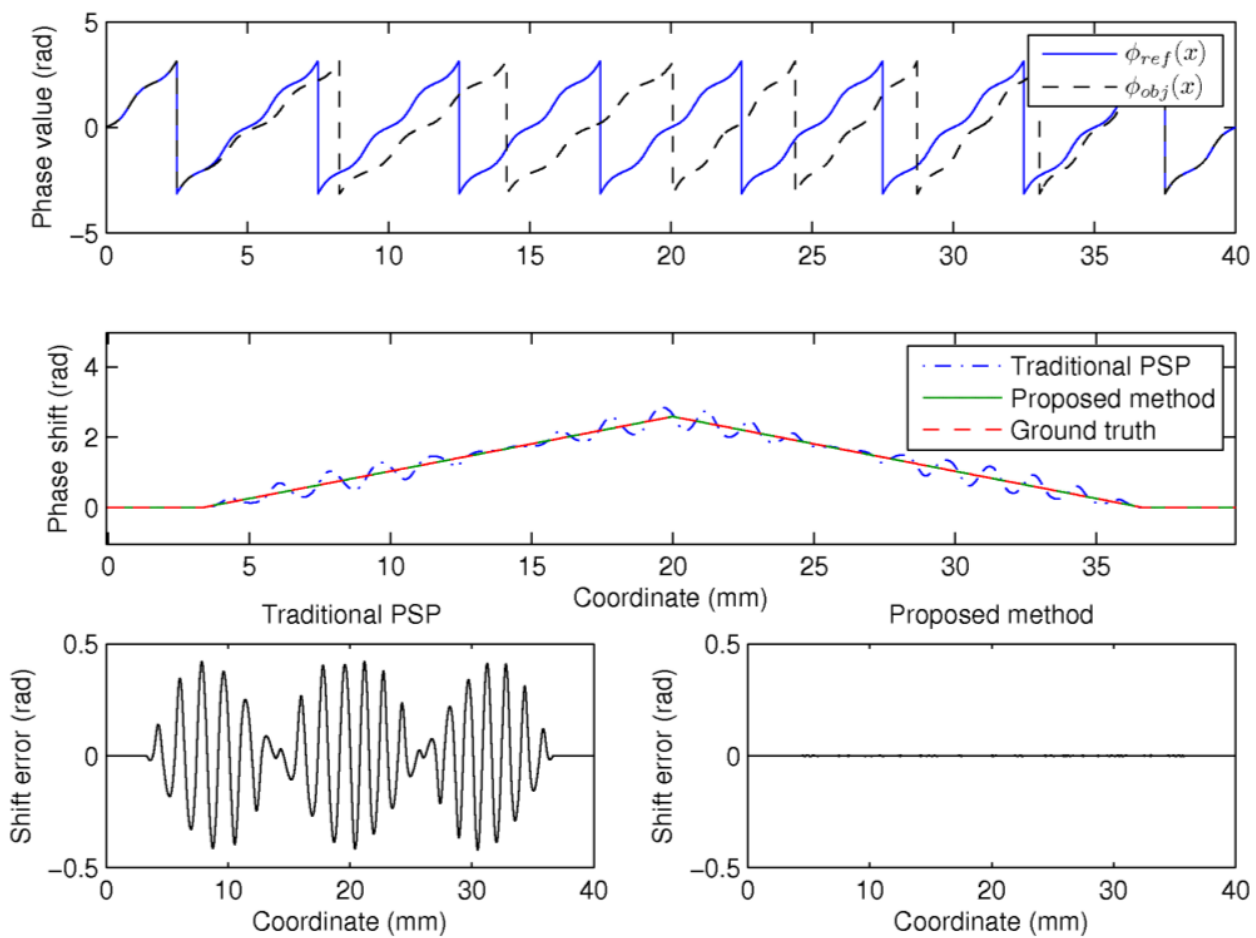

Figure 3. A cross section of simulation results, where $\gamma=2.1, \delta(x)=-0.015|x-20|+0.25$ and $f=0.2 \mathrm{~mm}^{-1}$. Top: distorted phase values before and after adding shift. Mid: calculated values and true value of phase shift caused by the object. Bottom: comparison of errors

The simulation results show that the shift of distorted signal can be calculated with very small errors. To verify it further, we apply the proposed method in practical experiments. Our experiment employs a HITACHI CP-X260 projector and a DuncanTech MS3100 camera (see Figure 4) to generate and capture fringe patterns respectively. And gamma parameter of the projector is estimated between 2 and 3. Parameters shown in Figure 1: $D=1442 \mathrm{~mm}$ and $L=345 \mathrm{~mm}$. A white triangular model is chosen as the first object. As shown in Figure 5, shift based PSP has significantly reduced the error in 3 -step PSP and RMSE drops from 0.194 to 0.034 . We also test the proposed method by measuring a more complicated object, a plaster cast in the shape of a hand as shown in Figure 4. Figure 6 compares results calculated in different ways. The reconstructed surface is much smoother by shift based three-step PSP, which confirms that the proposed method can successfully correct the error from gamma distortion. 
a

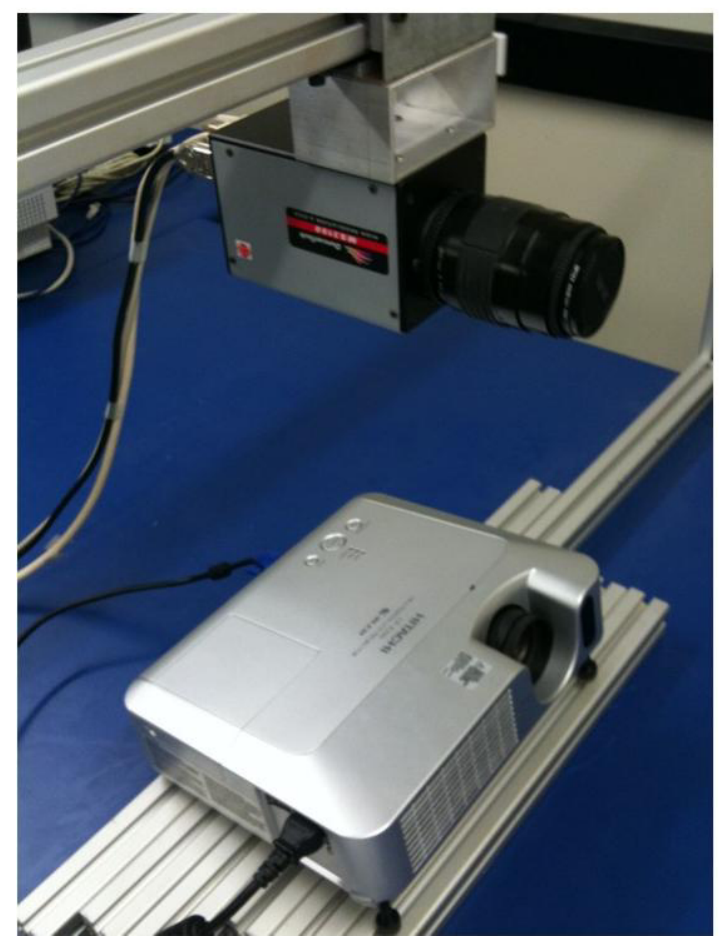

b

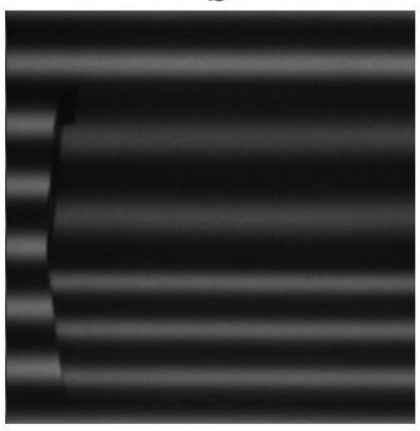

C

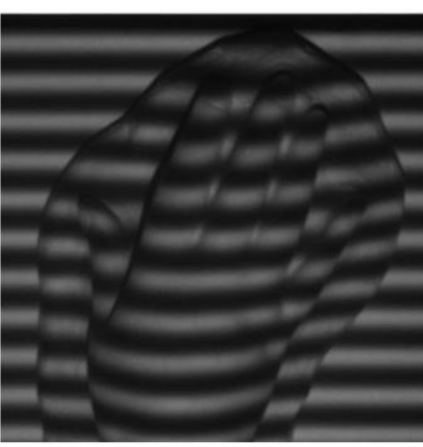

Figure 4: a: optical instruments. b: the triangular shape object. c: the complicated object used in experiment.
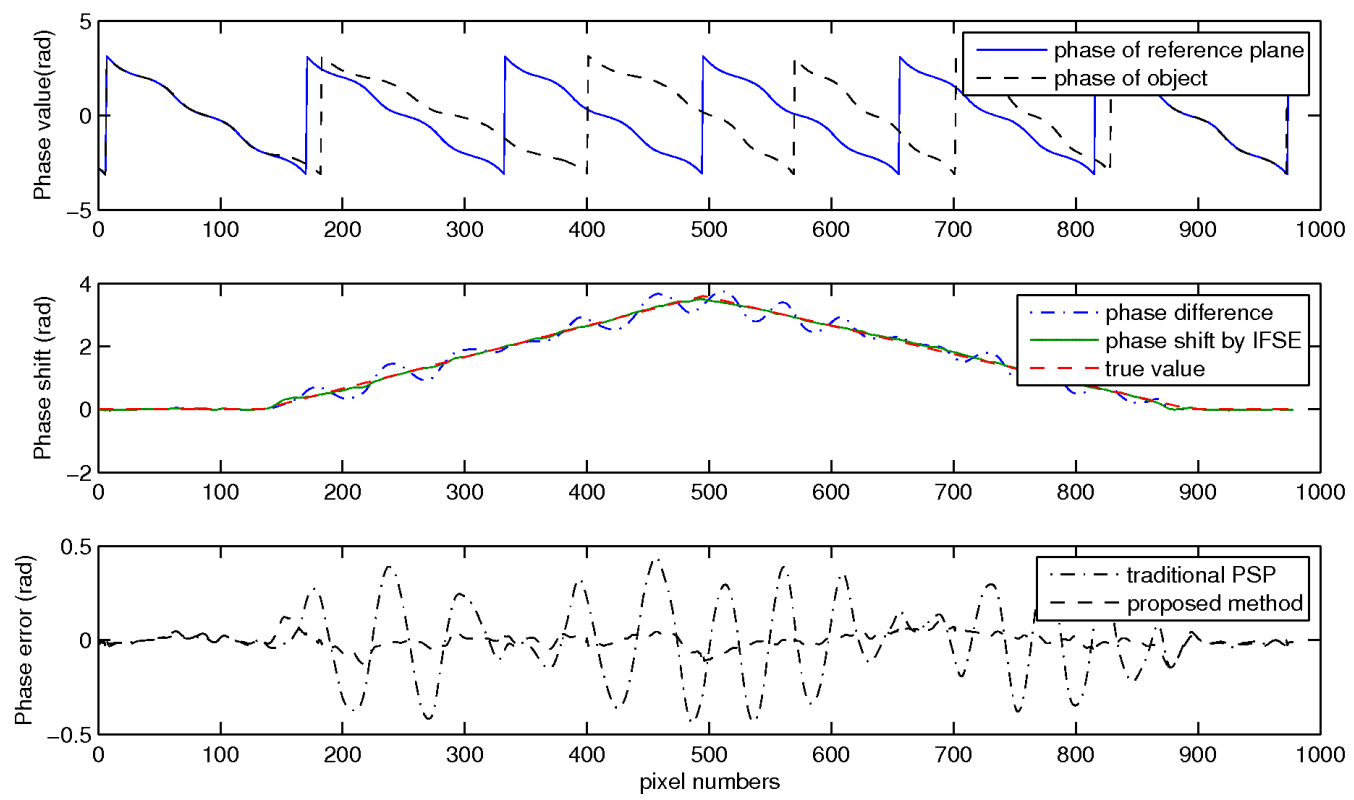

Figure 5. Experiment results of a simple object. Top: phase values on the reference plane and the object. Mid: calculated values and true value of phase shift caused by the object. Bottom: comparison of errors. 

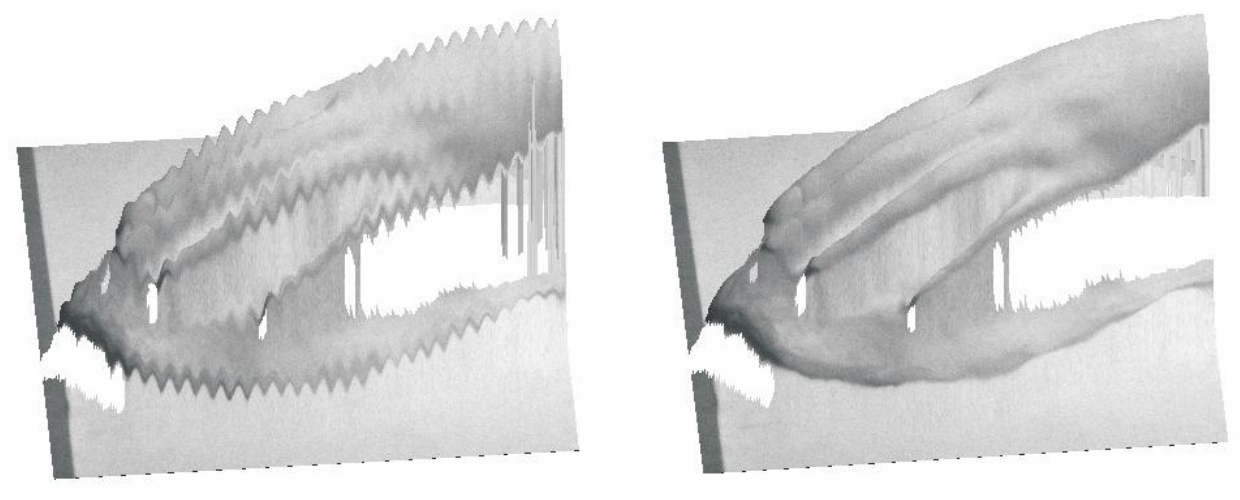

Figure 6. Experiment results of a complicated object. Left: result of traditional 3-step PSP. Right: result of shift based on 3-step PSP.

\section{CONCLUSION}

In this paper, we have introduced a novel method to correct errors by Digital video projector in three-step PSP, which is based on calculating phase shift between a reference plane and objects. The effectiveness of this method has been demonstrated by both computer simulation and experiments; the error caused by projectors' gamma nonlinearity has been greatly reduced. Because we employ IFSE to calculate the phase shift, the proposed method does not require any additional equipment and is automatic and easy to implement.

\section{REFERENCE}

[1] M. Takeda, H. Ina, and S. Kobayashi, "Fourier-transform method of fringe-pattern analysis for computer-based topography and interferometry," Journal of the Optical Society of America, 72(1), 156-160 (1982).

[2] M. Takeda, and K. Mutoh, "Fourier-transform profilometry for the automatic-measurement of 3-d object shapes," Applied Optics, 22(24), 3977-3982 (1983).

[3] V. Srinivasan, H. C. Liu, and M. Halioua, "Automated phase-measuring profilometry of 3-d diffuse objects," Applied Optics, 23(18), 3105-3108 (1984).

[4] X. Y. Su, W. S. Zhou, G. Vonbally et al., "Automated phase-measuring profilometry using defocused projection of a ronchi grating," Optics Communications, 94(6), 561-573 (1992).

[5] S. Toyooka, and Y. Iwaasa, "Automatic profilometry of 3-d diffuse objects by spatial phase detection," Applied Optics, 25(10), 1630-1633 (1986).

[6] Y. Hu, J. Xi, J. F. Chicharo et al., "Inverse function analysis method for fringe pattern profilometry," IEEE Transactions on Instrumentation and Measurement, 58(9), 3305-3314 (2009).

[7] M. J. Baker, J. Xi, and J. F. Chicharo, "Neural network digital fringe calibration technique for structured light profilometers," Applied Optics, 46(8), 1233-1243 (2007).

[8] P. S. Huang, Q. J. Hu, and F.-P. Chiang, "Double three-step phase-shifting algorithm,” Applied Optics, 41(22), 4503-4509 (2002).

[9] S. Zhang, and P. S. Huang, "Phase error compensation for a 3-d shape measurement system based on the phase-shifting method," Proc. SPIE 6000, 60000E-10.

[10] S. Zhang, and S.-T. Yau, "Generic nonsinusoidal phase error correction for three-dimensional shape measurement using a digital video projector," Applied Optics, 46(1), 36-43 (2007).

[11] K. Liu, Y. Wang, D. L. Lau et al., “Gamma model and its analysis for phase measuring profilometry,” J. Opt. Soc. Am. A, 27(3), 553-562 (2010).

[12] Y. Hu, J. Xi, Z. Yang et al., "Study on generalized analysis model for fringe pattern profilometry," IEEE Transactions on Instrumentation and Measurement, 57(1), 160-167 (2008). 